\title{
Computation of Separation Ahead of Blunt Fin in Supersonic Turbulent Flow
}

\section{Ching-mao Hung}

(NASA-TM-89410) COMEOTATICN CF SEPAKATION

AEEAD OF BLUNT FIN IN SUPEESCAIC TURBULENT

FLOW (NASA) $9 \mathrm{p}$ CSCL $01 A$

N87-16791

Unclas

G3/02 43733

December 1986 


\section{Computation of Separation Ahead of Blunt Fin in Supersonic Turbulent Flow}

Ching-mao Hung, Ames Research Center, Moffett Field, California

December 1986

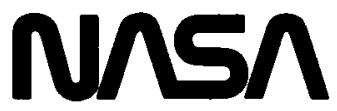

National Aeronautics and

Space Administration

Ames Research Center

Moffett Field, California 94035 


\title{
COMPUTATION Of SEPARATION AHEAD of BLUNT FIN
}

\section{in SUPERSONIC TURBULENT FLOW}

\author{
Ching - mao Hung \\ NASA Ames Research Center, Moffett Field, CA, USA
}

\section{INTRODUCTION}

Supersonic flow over a protuberance mounted on a surface (Fig. 1) presents a typical problem of an interaction of a three-dimensional shock wave and a boundary layer. The bow shock causes the boundary layer to separate from the surface ahead of the obstacle, resulting in a separated flow region composed of horseshoe vortices near the surface and a lambda-type shock pattern ahead of the protuberance. The shock wave emanating from the separated flow region (separation shock) impinges on the bow shock, and causes intense heating and high pressure locally around the leading edge of the protuberance.

For a hemicylindrical blunt fin, it has been observed from experiments (e.g., Refs. 1-2) and numerical simulations ${ }^{[3]}$ that many of the important flow length scales are primarily dependent on the diameter of the fin leading edge, $D$, and only weakly dependent on the free-stream Mach number, $M_{\infty}$, and properties of the incoming boundary layer. Variation of the thickness of incoming boundary layer from $\delta / D=1.0$ to 0.1 in Ref. 3 produced approximately the same size of horseshoe vortex and therefore the spatial extent of the interaction. The length of separation along the line of symmetry is about 2.0 to $2.5 \mathrm{D}$, provided the incoming boundary layer is turbulent. As the bluntness of the leading edge increases, one can expect the length scales, such as the length of separation, to increase. The question is how much it will increase.

In this paper we investigate numerically the separation ahead of a flat-faced blunt fin in a supersonic turbulent boundary layer. The flow field is calculated for a free-stream Mach number of 4.9 and unit Reynolds number of $52 \times 10^{6} \mathrm{~m}^{-1}$, and the results are compared with the experiment conducted by Rodi and Dolling ${ }^{[4]}$. The question of length of separation is discussed first. With all of the flow-field data at hand, we can study details of the flow structure, such as the existence of secondary separation and the horseshoe vortex.

\section{NUMERICAL PROCEDURE}

The governing equations of the present analysis are the time-dependent, compressible Navier-Stokes equations incorporating the thin-layer approximation by Baldwin and Lomax ${ }^{[5]}$. A numerical procedure developed by Hung and Kordulla $^{[6]}$ is used. The basic numerical scheme is MacCormack's ${ }^{[7]}$ explicitimplicit predictor-corrector algorithm with a Strang-type ${ }^{[8]}$ time-splitting. An 
algebraic eddy-viscosity turbulence model by Baldwin and Lomax ${ }^{[5]}$, with a "modified distance ${ }^{n[3]}$, is used to close the system of equations. Details of the numerical technique, mesh system, boundary conditions and turbulence model are discussed in Refs. 3 and 6.

\section{RESULTS AND DISCUSSION}

Figure 2 shows a half-C-type mesh system of $40 \times 32 \times 32$ points for a flat-faced blunt fin on a flat plate. The flow to be simulated has free-stream conditions of $M_{\infty}=4.90$, and unit Reynolds number of $52 \times 10^{6} \mathrm{~m}^{-1}$. The fin has the thickness $D=0.64 \mathrm{~cm}$ and is placed $26.7 \mathrm{~cm}$ from the leading edge of the flat plate. which results in an incoming boundary-layer thickness of $0.52 \mathrm{~cm}$. Figure 3 shows the comparison of surface pressure along the symmetry line. (The plot of u-velocity at the point of $\mathrm{k}=2$ is also shown for later discussion.) The agreement is reasonably good, considering the coarseness of the grid points used near the point of separation.

\section{Length of Separation}

One of the quantities of primary interest in a protuberance and boundary layer interaction is the length of separation. It is very often used with some empirical correlation to estimate the peak pressure and heat transfer on the leading edge of blunt fin. To understand the effect of the flat-faced fin on the length of separation, let us make a simple conjecture. As sketched in Fig. 4, the length of separation is about 2.5 D on the line of symmetry for a hemicylindrical blunt fin. As the hemicylindrical portion is removed, the bow shock will follow as sketched. Intuitively, one would expect that the location of separation should stay roughly the same. That means the length of separation would increase to about 3.0 D, or a little less. Surprisingly, it increases even more (by a factor of about two). The length of separation is about 5.2 D for the present computation and about 5.5 D experimentally ${ }^{[4]}$. This can be seen clearly in the plots of simulated "oil flow" on the flat plate (Fig. 5). The calculated results confirm the observation from experiments ${ }^{[4,9]}$ of this drastic increase in separation length for flow over a flat-faced fin.

\section{The Existence of Secondary Separation}

In Ref. 4, the measured pressure shows the appearance of a small second pressure peak (or a "kink"), which was interpreted as a sign of the existence of a secondary separation and the development of an additional pair of vortices. Another question arises with this appearance of a secondary separation. Whenever there is a secondary separation, the issue is whether the main horseshoe vortex will remain as one or bifurcate into two vortices. Sedney and Kitchens ${ }^{[10]}$ suggest that the main horseshoe vortex will bifurcate and that there will be an even number of vortices. The process starts with two vortices - one main horseshoe vortex and one small one near the corner as shown in Fig. 9 of Ref. 6. As a secondary 
separation appears, the main horseshoe vortex bifurcates, and the vortices develop by pairs (an even number, as shown in Fig. 11 of Ref. 10). For certain flow conditions, the existence of as many as six vortices has been inferred from experimental oil flow. Now, with the complete flow-field information from calculations available, we can examine the flow field closely and perhaps can strengthen (or weaken) the arguments used in such inferences.

Figure 6 shows calculated particle paths in the plane of symmetry. The horseshoe vortex does indeed bifurcate into two vortices of the same sign. However, there is no secondary separation under the main horseshoe vortices, and there is an odd number of vortices - three in this case. The kink of static pressure corresponds to a deceleration of the reverse u-velocity (see Fig. 3). Under different flow conditions, the deceleration of reverse u-velocity eventually may lead to a secondary separation. This means that the appearance of a kink in pressure is only a necessary condition for the existence of secondary separation, but not a sufficient condition. Even though they might interact with each other, the secondary separation is generally controlled by the pressure field, rather than vice versa.

A drastic change of velocity leads to a drastic change of skin friction which might result in a situation of temporary accumulation of oil flow on the surface. (In the present case, if the oil flow were to reach a "steady-state", there would be only one oil-accumulation line remaining, the outermost one.) Since the experimental oil flow may not have reached a steady state, an accumulation of oil flow on the surface is not necessarily a line of separation (as is usually inferred). This argument has arisen in previous experiments and calculations for different geometries ${ }^{[1]}$.

\section{Structure of the Horseshoe Vortex}

It has been generally believed that the structure of horseshoe vortices is in accord with the jet-maze model of Norman ${ }^{[12]}$ (as sketched in Fig. 7a). The separation point is connected to the second vortex (indicated as 2 ). The fluid between $a-b$ and $c-d$ (indicated as 2) is entrained in the second vortex, between b-c (indicated as 1) in the first vortex, and between d-e (indicated as 3) in the third vortex near the corner. The fluid above e remains outside the vortices. The structure based on the present calculations (Fig. 6) is sketched in Fig. 7b. The separation point ties with the first vortex and the four "layers" of fluid entrained in the three vortices are now marked 1-2-1-3, instead of 2-1-2-3 as in Fig. 7a. There is a saddle point between the first and second vortex. Under the saddle point, the reverse velocity slows down ( see Fig. 3) and there is a kink in the surface pressure.

Evidently, the evolution from one to two vortices starts from the lower side of the vortex because of the confrontation of an adverse pressure gradient in the lower-side reversed flow. Such a feature can be observed in the computational result presented in Ref. 3, as the boundary-layer thickness decreases from $\delta / D=$ 
1.0 to 0.26 . The horseshoe vortex starts to show a "pinch" and there is a kink in pressure (see Figs. 13a and 14c in Ref. 3).

Since the two vortices are of the same sign, they have a tendency to rotate about each other and roll up. Indeed, the present result (Fig. $7 \mathrm{~b}$ ) is simply the jet-maze model (Fig. 7a) with vortices 1 and 2 twisted together $180^{\circ}$ in the direction of the vortices. We believe that a flow structure of further twisting as sketched in Fig. 8 is possible. As the fluid flows far downstream, one might expect that the vortices would roll up and eventually merge to become one (as occurs in trailing vortices). However, in the present domain of computation, there seems to be no further roll-up of the two vortices as they convect downstream.

\section{CONCLUSION AND REMARKS}

Separation ahead of a flat-faced blunt fin in a supersonic turbulent boundary layer was studied numerically. The following observations and conclusions were made.

(a) The length of separation increases to about 5.2 D, compared with about 2.0 to $2.5 \mathrm{D}$ for the typical hemicylindrical results, and this numerical result confirms the experimental observations.

(b) Even though there is a "kink" in pressure in the present case, there is no secondary separation under the main horseshoe vortices and there are three vortices; it is therefore concluded that the number of vortices is not always an even number.

(c) For the case investigated the separation point is connected to the inner (first) horseshoe vortex, rather than the outer (second) one. The four layers of fluid marked 1-2-1-3 as in Fig. $7 \mathrm{~b}$ entrain in the three vortices respectively.

\section{REFERENCES}

[1]. Kaufman, L.G., Korkegi, R.H., and Morton, L.C. "Shock Impingement Caused by Boundary Layer Separation Ahead of Blunt Fin," ARL 72-0118, 1972, Wright-Patterson Air Force Base, Ohio.

[2]. Dolling, D.S. and Bogdonoff, S.M. "Blunt Fin-Induced Shock Wave/ Turbulent Boundary Layer Interaction," AIAA J., Vol. 20, No. 12, 1982, pp. 1674-1680.

[3]. Hung, C. M. and Buning, P. G., "Simulation of Blunt-Fin-Induced Shock Wave and Turbulent Boundary-Layer Interaction," J. Fluid Mechanics, Vol. 154, May 1985, pp.163-185

[4]. Rodi, P. E. and Dolling, D. S. “ Experimental Study of the Effects of Leading Edge Geometry on Fin Induced Turbulent Separated Flow at Mach 5: Preliminary Results," AIAA Paper 86-0344, Jan. 1986.

[5]. Baldwin, B.S. and Lomax, H. "Thin-Layer Approximation and Algebraic Model for Separated Turbulent Flows," AIAA paper 78-257, Jan. 1978. 
[6]. Hung, C. M. and Kordulla, W., "A Time-Split Finite-Volume Algorithm for Three-Dimensional Flowfield Simulation,” AIAA J., Vol. 22, No. 11, 1984, pp. 1564-1572.

[7]. MacCormack, R.W. "A Numerical Method for Solving the Equations of Compressible Viscous flow," AIAA J., Vol. 20, No. 9, 1982, pp. 1275-1281.

[8]. Strang, G. "On the Construction and Comparison of Difference Schemes," SIAM J. Numerical Analysis, Vol. 5, 1968, pp. 506-517.

[9]. Saida, N. "Separation Ahead of Blunt Fins in Supersonic Turbulent Boundary Layers," Presented at IUTAM Symposium on Turbulent Shear Layer/ Shock Wave Interaction," Palaiseau, France, Sept., 1985.

[10]. Sedney, R. and Kitchens, C. W.,Jr. "The Structure of Three-Dimensional Flows in Obstacle-Boundary Layer Interaction," Flow Separation, AGARD CP-168, paper 37, 1975.

[11]. Hung, C.M., "Computation of Three-Dimensional Shock Wave and Boundary Layer Interactions," NASA TM-86780, August 1985.

[12]. Norman, R.S. “On Obstacle Generated Secondary Flows in Laminar Boundary Layers and Transition to Turbulence," Ph.D. Thesis, Illinois Inst. Technol., 1972.

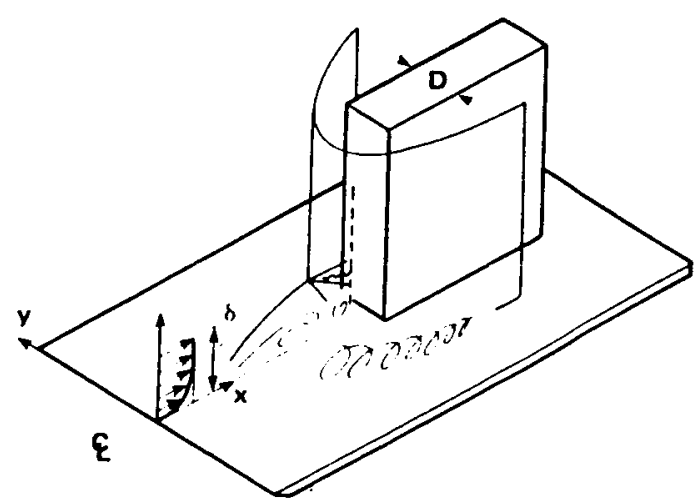

Fig. 1 Supersonic flow over a blunt fin on a flat plate.

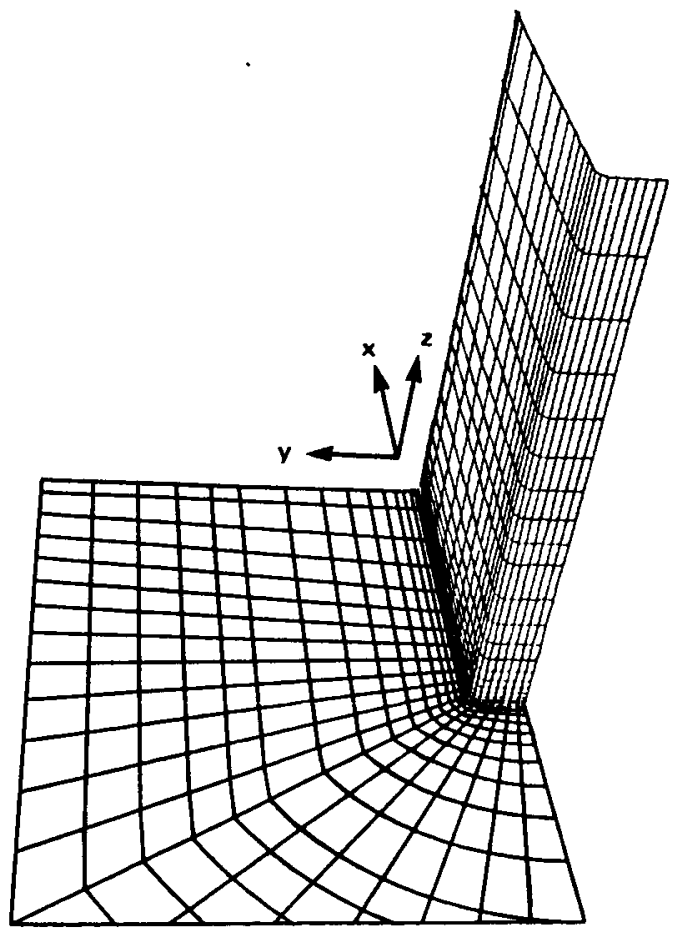

Fig. 2 Mesh system. 


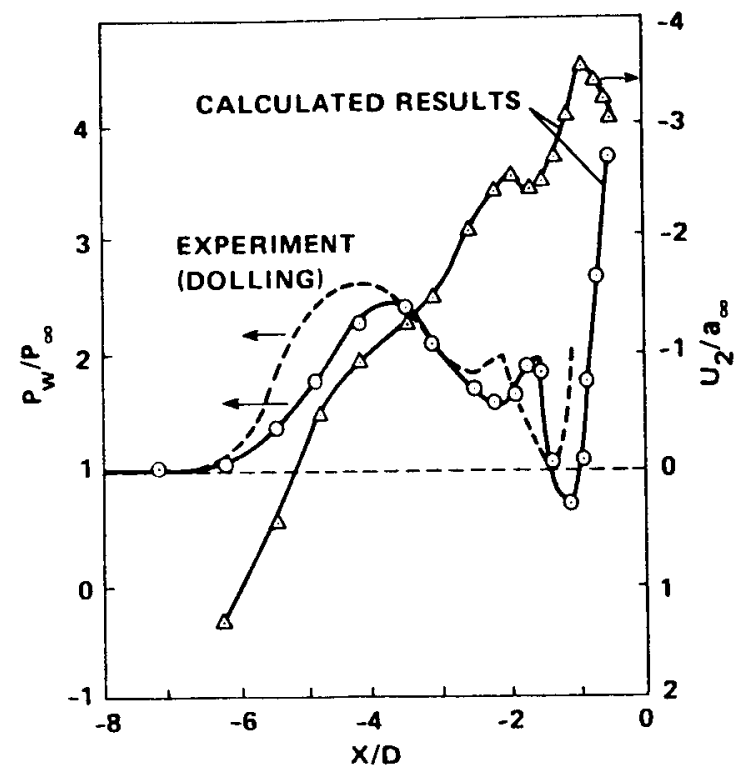

Fig. 3 Comparison of pressure, and plot of u-velocity at $k=2$ along line of symmetry.

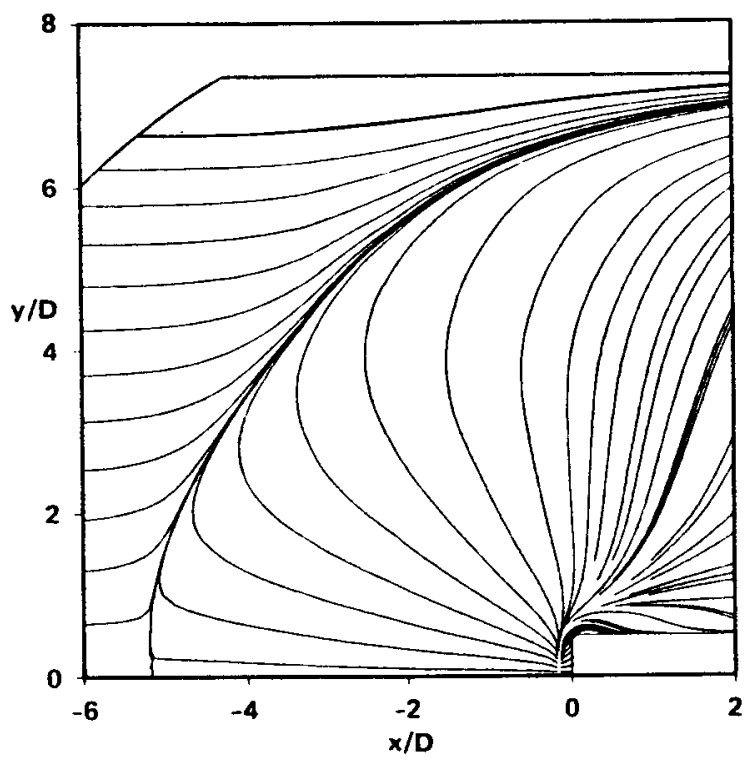

Fig. 5 Particle paths in the horizontal plane $(k=2)$.

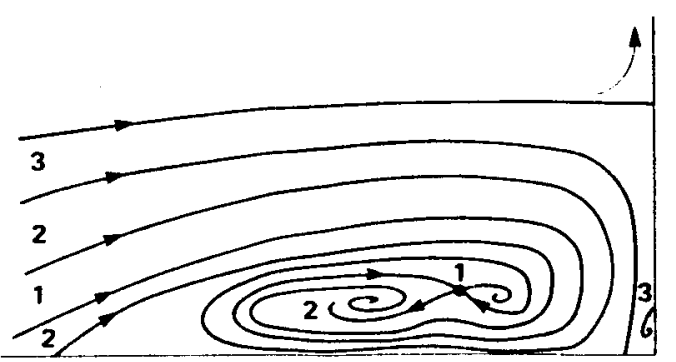

Fig. 8 Horseshoe vortices with a further twist.

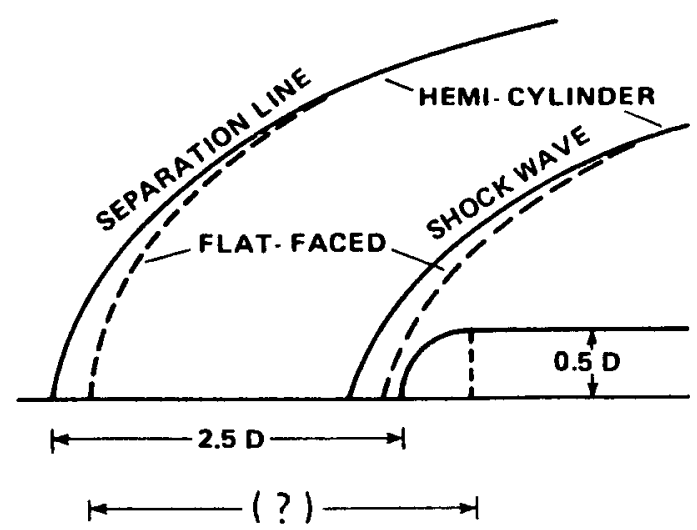

Fig. 4 A sketch of separation line and shock wave for varying bluntness.

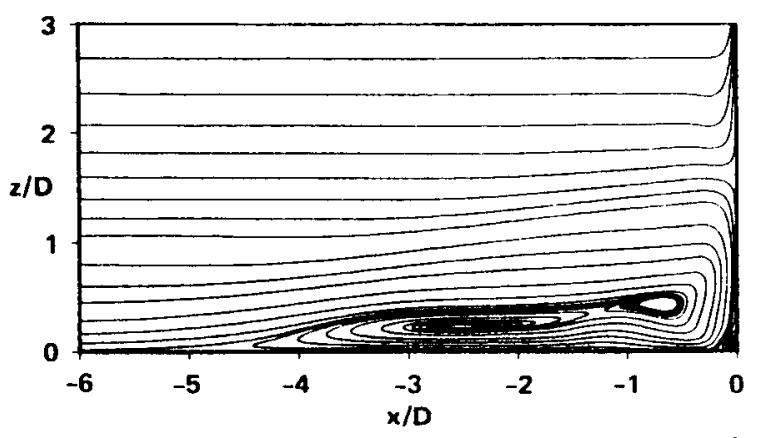

Fig. 6 Particle paths in the plane of symmetry.

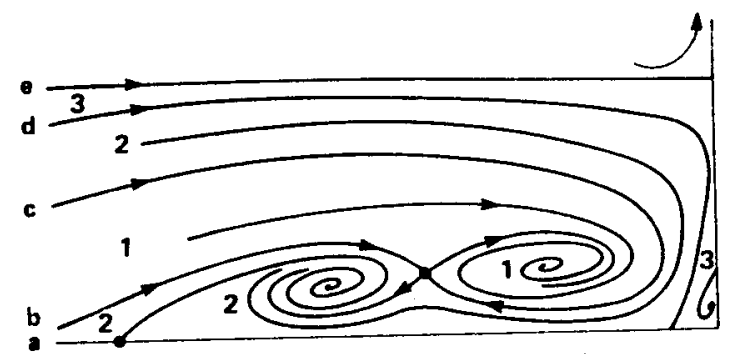

a. Jet-maze model (Norman).

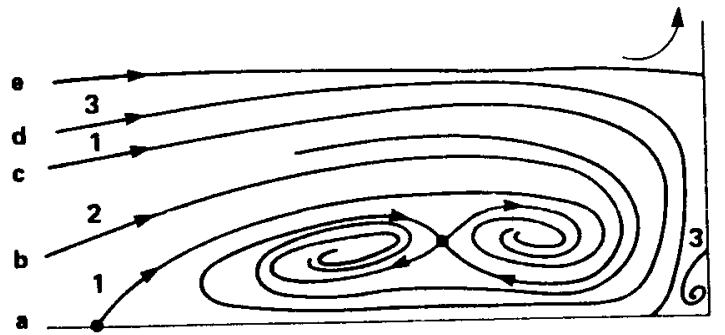

b. Model based on present result.

Fig. 7 Structure of llorseshoe vortices. 


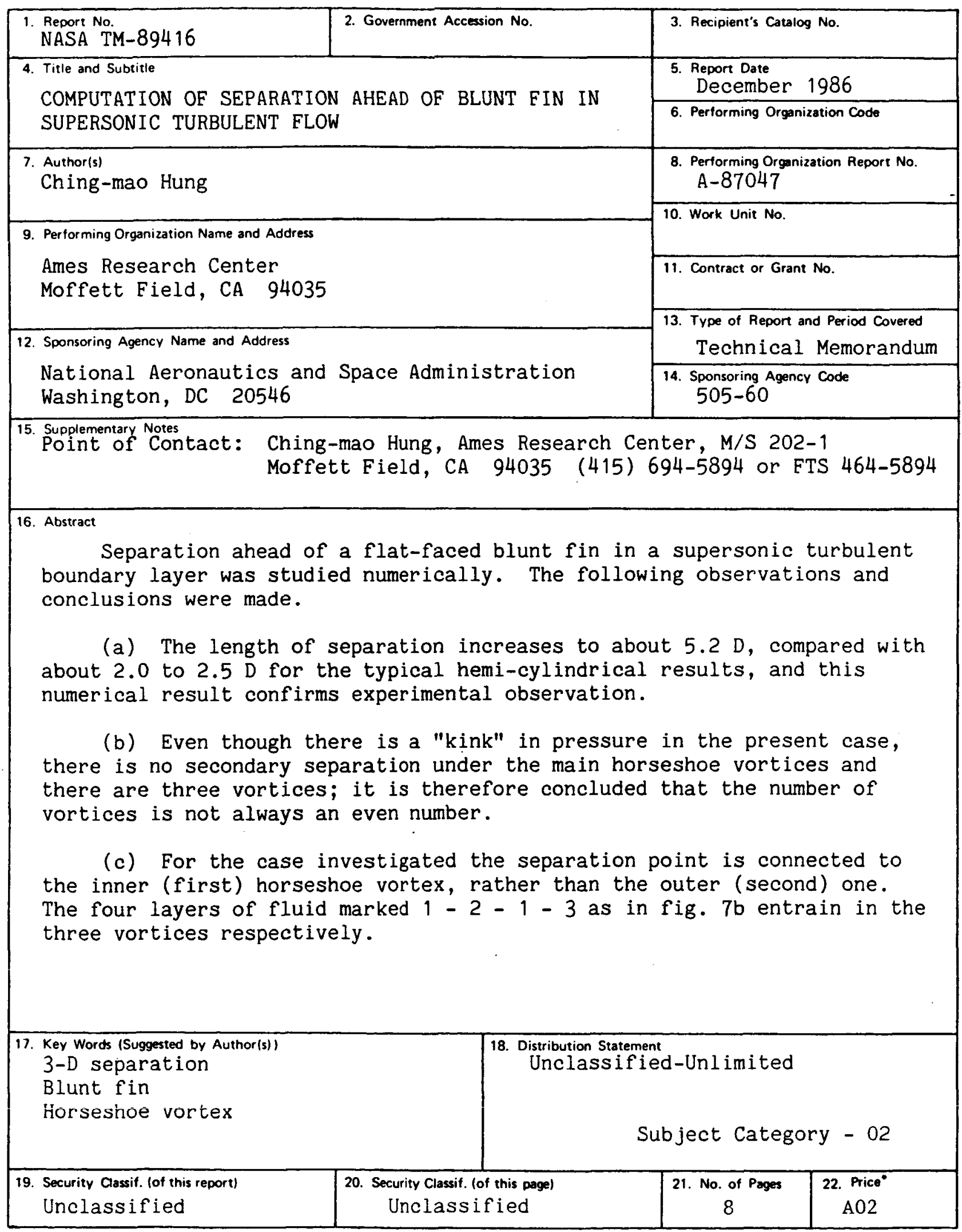

- For sale by the National Technical Information Service, Springfield, Virginia 22161 